\title{
Editors Letter
}

\author{
Eva Johansson
}

Published online: 18 February 2011

(C) Springer Science+Business Media B.V. 2011

Dear readers

From this year we are proud to be able to publish three issues of the International Journal of Early Childhood and to do this every year. To keep the high scientific level of the published papers and to increase the amount of submitted and published papers is an exciting and encouraging duty for the editorial board as well as for our readers and authors. We therefore invite all of you to submit original research on and with children, on childhood and early childhood education across various social and cultural contexts!

We are also happy to inform you that the editorial board has extended its expertise. We very much welcome Professor Susan Wright and Professor Nirmala Rao to the editorial board. Susan Wright is a Professor of Education at the National Institute of Education, Nanyang Technological University, Singapore. She is an arts educator involved in research that focuses on children's learning using artistic symbol systems. Nirmala Rao is Professor, at the Faculty of Education, University of Hong Kong. She is a Developmental and Chartered (Educational) Psychologist, who has published widely on early childhood development and education in Asia.

In this issue of the International Journal of Early Childhood we are pleased to present four interesting articles of various types.

Two of these concern gender issues in Early Childhood Education. Both authors refer their research to discourses and possible subject positions in relation to gender in the context of Swedish preschools.

The first article Becoming a preschool child: Subjectification in toddlers during their introduction to preschool, from a gender perspective is written by Annika Månsson concern the subject positions that can be adopted by children during their introduction to preschool and how gender forms part of this positioning. Data is

E. Johansson $(\bowtie)$

Department of Early Childhood Education, Faculty of Arts and Education, University of Stavanger,

4036 Stavanger, NO, Norway

e-mail: eva.johansson@uis.no 
obtained by observations of interactions between teachers and children. The analysis implies various children's positions connected with diverse situations and persons, allowing openings in the categories of 'girl' and 'boy'. The position of 'being competent' for example, can be attributed to both girls and boys. The position of 'being needy' on the contrary is ambiguous from a gender perspective. When girls for example take up space, and make demands, they are positioned as obstinate rather than being viewed as being needy.

The second article Constructions of girls in preschool parent-teacher conferences by Ann-Marie Markström and Maria Simonsson is based on audio taped conferences where teacher and parents meet and talk about the child's life and wellbeing in preschool. The authors investigate the discursive constructions of girls and how girls are positioned, assessed and constructed by adults in the conferences. The analysis indicates that girls are positioned as 'ordinary preschool girls', doing what preschool girls are expected to do, and as 'girlish girls' associated with behaviour and characterisations embedded in a historically feminine discourse. Markström and Simonsson also identify discourses that challenge and encourage different femininities including one that draws on masculinity and is conceived as empowering.

The two other articles in this issue of the International Journal of Early Childhood addresses the context of Early Childhood Education and Early School and uses cross-cultural and longitudinal approaches for their studies.

The article A multicultural perspective on play and learning in primary school by Ole Fredrik Lillemyr, Frode Søbstad, Kurt Marder and Terri Flowerday, takes a focus on indigenous and non-indigenous groups of children in the early years of school in Australia, USA and Norway. The intention was to identify cultural profiles among the students' groups on their interests in play, learning preference, selfconcept and motivational orientation, applying scales and interviews. The results revealed a tendency of indigenous students to favour a traditional, teacher-directed concept of learning, compared to non-indigenous students. In addition the results indicated a lower self-concept among indigenous students, compared to nonindigenous students. Friendship and sense of competence are, according to these authors, important for students' motivation to participate and achieve in school. A sense of relatedness is quintessential in this concern.

The final article in this issue: Synthèse d'une étude longitudinale portant sur les effets d'un programme de maternelle 4 ans à temps plein is written by Claire Maltais, Yves Herry, Isabelle Émond and Catherine Mougeot, The authors present a longitudinal study evaluating a full-day educational program to all 4-year-old children in the territory of Ontario, Canada. The key question in the study concerns: Is a full-day preschool program more likely to enhance, in the longer term, linguistic, academic, social-emotional, and psychomotor development than a halfday preschool program? The results indicate that the full-day preschool program for 4-year-old children is more likely to enhance, in the longer term, linguistic and academic development in reading and mathematics. On the contrary, the program indicated little effect on writing competence, and in regard to social-emotional and psychomotor development. 
Dear readers and authors-hopefully you will be inspired by these various articles and topics all of significant importance in early childhood. Please follow up and continue the critical discussion and the development of knowledge in the field of early education.

Göteborg 2011-01-19

Eva Johansson Editor

Chers lecteurs et lectrices,

Nous annonçons avec fierté qu'à compter de cette année, nous sommes en mesure de publier trois numéros de la Revue internationale de l'enfance préscolaire, par année. Maintenir un haut niveau scientifique et augmenter le nombre de textes soumis et publiés est une tâche stimulante et encourageante tant pour le comité de rédaction que pour nos lecteurs et nos auteurs. Vous êtes donc tous invités à soumettre vos recherches originales sur et avec les enfants, sur l'enfance et sur l'éducation préscolaire dans divers contextes sociaux et culturels!

Il nous fait également plaisir de vous apprendre que l'expertise du comité de rédaction s'est élargie. Nous souhaitons la plus chaleureuse bienvenue à professeure Susan Wright et à professeure Nirmala Rao au comité de rédaction. Susan Wright est professeure d'éducation au National Institute of Education, Nanyang Technological University, Singapour. C'est une spécialiste en éducation artistique impliquée dans des recherches centrées sur l'apprentissage des enfants par l'utilisation de systèmes symboliques artistiques. Nirmala Rao est professeure à la faculté d'Éducation de l'Université de Hong Kong. Elle est psychologue du développement, experte en éducation et a largement publié sur le développement et l'éducation préscolaire en Asie.

Ce numéro de la Revue internationale de l'enfance préscolaire présente quatre intéressants articles de divers types.

Deux d'entre eux portent sur les questions de genre en éducation préscolaire. Les deux auteurs relient leurs travaux aux discours et aux positions de sujet liées au genre possibles dans le contexte préscolaire suédois.

Le premier article Becoming a preschool child: Subjectification in toddlers during their introduction to preschool, from a gender perspective, d'Annika Månsson, traite des positions de sujet que les enfants peuvent adopter pendant leur initiation à la maternelle et comment le genre fait partie de ce positionnement. Les données sont obtenues par l'observation des interactions entre les enseignantes et les enfants. L'analyse implique les différentes positions des enfants en lien avec diverses situations et personnes et permettant des ouvertures dans les catégories de «fille» et «garçon». La position de «compétent», par exemple, peut être attribuée tant aux filles qu'aux garçons. La position d' «ayant besoin», au contraire, est ambiguë d'un point de vue de genre. Par exemple, quand les filles occupent l'espace et font des demandes, elles sont positionnées comme «obstinées» plutôt que comme «ayant besoin».

Le deuxième article, Constructions of girls in preschool parent-teacher conferences, par Ann-Marie Markström et Maria Simonsson, porte sur les enregistrements audio de rencontres où l'enseignant et les parents parlent de la vie et du bien-être de l'enfant en maternelle. Les auteures se penchent sur les 
constructions discursives sur les filles et sur la façon dont les filles sont positionnées, évaluées et construites par les adultes dans ces rencontres. L'analyse indique que les filles sont positionnées comme des fillettes ordinaires qui font ce que l'on attend que fassent des fillettes du préscolaire, et comme de «vraies filles», associées aux comportements et caractérisations inscrits dans un discours féminin historique. Markström et Simonsson identifient aussi des discours qui défient et encouragent différentes féminités, y compris celle qui s'appuie sur la masculinité et qui est vue comme donnant du pouvoir

Les deux autres articles de ce numéro de la Revue internationale de l'enfance préscolaire portent sur le contexte de l'éducation préscolaire et la scolarisation précoce et s'appuient sur des approches transculturelles et longitudinales.

A multicultural perspective on play and learning in primary school, par Ole Fredrik Lillemyr, Frode Søbstad, Kurt Marder et Terri Flowerday, se penche sur des groupes d'enfants autochtones et non autochtones dans les premières années de scolarisation en Australie, aux États-Unis et en Norvège. Il a pour but d'identifier les profils culturels chez ces groupes d'élèves quant à leur intérêt pour le jeu, leurs préférences d'apprentissage, leur concept de soi et l'orientation de leur motivation, au moyen d'échelles et d'entrevues. Les résultats révèlent une tendance chez les élèves aborigènes à favoriser un concept d'apprentissage traditionnel, dirigé par l'enseignant, comparativement aux élèves non aborigènes. Selon les auteurs, l'amitié et le sentiment de compétence sont importants pour la motivation des élèves à participer et réussir à l'école. Un sentiment d'appartenance est quintessentiel à cet égard.

Le dernier article de ce numéro, Synthèse d'une étude longitudinale portant sur les effets d'un programme de maternelle 4 ans à temps plein, est écrit par Claire Maltais, Yves Herry, Isabelle Émond et Catherine Mougeot. Les auteurs présentent une étude longitudinale évaluant un programme éducatif à plein temps pour tous les enfants de quatre ans dans un district de l'Ontario, Canada. Un programme à plein temps est-il davantage susceptible d'améliorer, à long terme, le développement linguistique, scolaire, socio émotif et psychomoteur qu'un programme de demijournée? Voilà la question clé de l'étude. Les résultats indiquent que le programme à plein temps est susceptible d'améliorer le développement linguistique et scolaire en lecture et mathématiques. Au contraire, le programme a peu d'effet sur la compétence à écrire et sur le développement socio émotif et psychomoteur.

Chers lecteurs et auteurs, nous espérons que vous serez inspirés par ces divers articles et sujets, tous d'une grande importance en éducation préscolaire. Vous êtes invités à y donner suite en poursuivant le débat critique et le développement des connaissances dans le domaine de l'éducation de la jeune enfance.

Göteborg, le 19 janvier 2011

Eva Johansson, rédactrice

Estimados lectores

A partir de este años, estamos orgullosos de estar en condiciones para publicar tres artículos de la Revista Internacional de Educación Temprana y de realizar esto cada año. Mantener el alto nivel científico de los artículos publicados y aumentar el número de papers presentados y publicados, es una tarea emocionante y alentadora, 
tanto para la junta editorial, como para nuestros lectores y autores. Por lo tanto, ¡los invitamos a todos ustedes a presentar investigaciones sobre niños y con ellos mismos, sobre infancia y educación preescolar en variados contextos sociales y culturales!

También estamos muy contentos de informarles que la junta editorial ha aumentado su número de expertos. Le damos una gran bienvenida a los profesores Susan Wright y Nirmala Rao a la junta editorial. Susan Wright es una profesora sobre Educación, en el Instituto Nacional de Educación, en la Universidad Tecnológica de Nanyang, en Singapur. Ella es profesora de arte involucrada en la investigación enfocada en el aprendizaje de niños y niñas utilizando sistemas de símbolos artísticos. Nirmala Rao es docente, en la Facultad de Educación de la Universidad de Hong Kong. Ella es Psicóloga en Desarrollo y (Educacional), que ha publicado ampliamente sobre desarrollo temprano y educación en Asia. She is a Developmental and Chartered (Educational) Psychologist,

En esta edición de la Revista Internacional de Educación Temprana estamos orgullosos de presentar cuatro interesantes artículos de diferentes tipos.

Dos de estos conciernen temas de género en la Educación Preescolar. Ambos autores refieren sus investigaciones a discursos y posibles posiciones del tema en relación al contexto de género en jardines infantiles Suecos.

El primer artículo Convirtiéndose en un niño preescolar: Significación, en menores, durante su introducción al preescolar, desde la perspectiva ha sido escrita por Annika Månsson, preocupándose por la postura del sujeto que puede ser adoptada por niños durante su introducción al preescolar, y cómo el género forma parte de este posicionamiento. La información es obtenida a través de observaciones e interacciones entre profesores y niños. El análisis implica la postura de varios niños conectada con diversas situaciones y personas, permitiendo aperturas en las categorías de "niña" y "niño". La postura de "ser competente", por ejemplo, puede ser atribuida a niños y niñas. La postura de "ser necesitado por otros", por el contrario, es ambigua desde ambas perspectivas de género. Por ejemplo, cuando las niñas toman un espacio y hacen demandas, se les posicione como obstinadas en vez de ser vistas como "que los otros las necesitan".

El segundo artículo Construcción del género femenino en niñas, en conferencias profesorespadres en el preescolar por Ann-Marie Markström y Maria Simonsson se basa en la grabación de audio de conferencias donde profesores y padres se conocen y hablan acerca de la vida de sus niños y de su bienestar en el preescolar. Las autoras investigaron la construcción discursiva de las niñas y su género, y de cómo ellas se posicionan, evaluadas y construidas por los adultos en las conferencias. El análisis indica que las niñas son posicionadas como "cualquier niña preescolar", haciendo lo que se espera que cualquier niña preescolar haga, y como "niñas femeninas", asociado con el comportamiento y las características desarrolladas históricamente en el discurso femenino. Markström y Simonsson también identificaron discursos que desafiaban y alentaban diferentes femeneidades, incluyendo algunas que se acercan a la masculinidad y se conciben como empoderamiento.

Los dos otros artículos en esta edición del Diario internacional de Educación Temprana incorporaron el contexto de Educación Preescolar y Escuela Primaria, y utilizaron acercamientos culturales longitudinales y cruzados para sus estudios. 
El artículo Una perspectiva multicultural sobre el juego y el aprendizaje en la escuela primaria por Ole Fredrik Lillemyr, Frode Søbstad, Kurt Marder y Terri Flowerday, tomaron como foco grupos de niños indígenas y no indígenas de los primeros años de la escuela en Australia, USA y Noruega. La intención era identificar perfiles culturales entre sus grupos de estudiantes sobre sus intereses en el juego, sus preferencias de aprendizaje, su auto-concepto y la orientación motivacional, aplicando escalas y entrevistas. Los resultados revelan una tendencia de los estudiantes indígenas hacia el concepto tradicional de aprendizaje con la guía del profesor, en comparación con los estudiantes no indígenas. La amistad y el sentido de competencia son, según estos autores, importantes en la motivación de los estudiantes a participar y generar logros en la escuela. Un sentido de relación se encuentra, por excelencia, en sus preocupaciones.

El artículo final de esta edición: Synthèse d'une étudelongitudinale portant sur les effets d'un programme de maternelle 4 ans à temps plein es escrito por Claire Maltais, Herry Yves, Isabelle Émond y Catherine Mougeot. Estos autores presentan un estudio longitudinal evaluando un programa educacional de día completo para todos los niños de cuatro años en el territorio de Ontario, Canadá. La pregunta clave en el tema del estudio: ¿Tiene el programa educacional de jornada completa más posibilidades de lograr, a largo plazo, el desarrollo lingüístico, académico, socialemocional y psicomotor que el programa de media jornada? El resultado indica que el programa de jornada completa para niños de cuatro años de edad sí posee más posibilidades de lograr, a largo plazo, el desarrollo lingüístico y académico en lectura y matemáticas. Por el contrario, el programa identifica bajos efectos en la competencia de escritura y en relación al desarrollo socioal-emocional y psicomotor.

Estimados lectores y autores - esperamos que encuentre inspiración en estos diferentes artículos y temas, todos de gran importancia en la educación temprana. Por favor sígannos y continúen la discusión crítica y el desarrollo del conocimiento en el campo de la educación temprana.

Göteborg 2011-01-19

Eva Johansson Editora 